\title{
Friedhof: Design
}

\author{
Erhard Taverna
}

Friedhöfe sind Endstationen, selbst wenn die Fahrt etwas länger dauert als früher. Aber auch am Ende gehört die Medizin zur Bürokratie kollektiver Regelungen von Entsorgung und Gedenken. Ohne Leichenschau keinen Totenschein und ohne diesen keine Bestattung. Im Zweifelsfall löst die Meldung beim Verhörrichter weitere Massnahmen aus: eine Legalinspektion oder die Obduktion im Rechtsmedizinischen Institut. In vielen Kantonen müssen kommunale Bestattungsreglemente von den Gesundheitsbehörden bewilligt werden. Ärzte sorgten im 19. Jahrhundert mit Argumenten der Hygiene für die Verlagerung der Totenäcker aus den Stadtzentren an die Peripherie. Die vorgeschriebenen fünf Aufbewahrungstage bis zur Bestattung erinnern an die Furcht, lebendig begraben zu werden, denn Geschichten über Scheintote waren im 18. und 19. Jahrhundert ein Massenphänomen. Diese Grabesfurcht, mit dem Fachbegriff Taphophobie, gehört auch heute noch zum medialen Gruselstandard. Wurden früher ausgeklügelte Sicherheitssärge entwickelt, so soll es heute Angehörige geben, die ein Handy mit in den Sarg plazieren. Wenn die Abschiedszeremonien noch im Gange sind, muss der Vibrationsalarm genügen, damit nicht ein eventuelles Klingeln die Abdankung stört. Ein Erfinder aus Osnabrück hat ein Mobiltelefon entwickelt und patentiert, das auch unter der Erde steten Empfang garantiert. Die wasserdichte Grabbeilage mit dem Namen «Engel-Telefon» ist etwa so gross wie ein Schuhkarton und kann nach 200 Stunden Abschiedsgesprächen belassen oder zurückgeschickt werden.

Die schleichende Abschaffung des Todes und der Wandel der Friedhöfe ist ein Thema der Ausstellung «Friedhof: Design, Objekte zwischen Ewigkeit und Vergänglichkeit» im Museum Bellerive an der Höschgasse in Zürich. Hölzerne Palette unterteilen die hellen Räume der herrschaftlichen Villa am See. Das gruftige Thema wird spielerisch-ernsthaft bewältigt, zeigt Friedhofsreglemente, Grabmale, Särge, Urnen und Grabschmuck, wie Totenhemd, Karten- und Brettspiele, Fachliteratur und Internetanzeigen. Friedhöfe wurden schon oft als Visitenkarten einer Gesellschaft beschrieben. Den Ausstellern ist es gelungen, eine Kulturgeschichte am Wandel der Konzepte und Positionen anregend und vielschichtig darzustellen. Der Vergleich alter

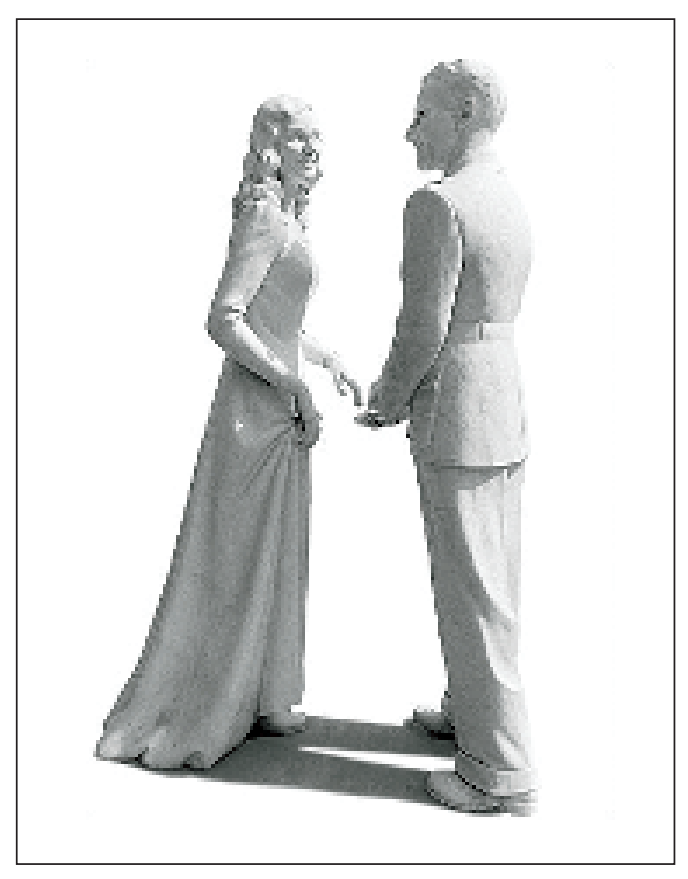

mit neuen Anlagen zeigt eine veränderte Friedhofsarchitektur, die den neuen Ritualbedürfnissen einer modernen Gesellschaft $\mathrm{zu}$ genügen sucht. Reichte bisher die Auswahl zwischen Erdbestattung, Kremation oder Gemeinschaftsgrab, sorgen heute zunehmend Baumbestattungen, Friedwälder, Heimurnen und virtuelle Gedenkstätten für Konkurrenz. Särge sind über Internet als einfaches Do-it-yourself-Modell oder bizarre Kunstkreationen, Urnen kompostierbar, gewässerschutzkonform oder als getöpferter Minigarten zu bestellen. Dass noch nie alle im Grabe gleich waren, demonstrieren die vielen Abbildungen und Modelle traditioneller Grabmale aus zahlreichen Ländern. Aussenseiter und Eliten zelebrieren wie immer die Mythen der Gegenwart. Sie lassen sich als Nekronauten ins All schiessen, im Tiefkühlklub Alcor einfrieren oder immateriell auf einer Homepage als interaktives Cybermausoleum rekonstruieren. Sie demonstrieren aufs neue, dass der Umgang mit dem eigenen Sterben zivilisatorisch geprägt ist. Die individualisierte Gesellschaft sucht nach kreativen Fluchtmöglichkeiten vor dem, was sie als behördlichen Friedhofszwang und einengende Normierung empfindet. Auf den medizinischen Wissenschaften gründen alle Utopien zukünfti- 
ger Heilungen und ewigen Lebens. Wo das Jenseits zur Fortsetzung des Diesseits wird, markiert der Tod nur noch eine technisch überwindbare Grenze. Die Zeit soll das Zeitliche aufheben. Was werden zukünftige Archäologen in unseren Nekropolen freilegen? Grabräuber werden die Orte des Todes meiden, denn was sie finden, ist medizinischer Sondermüll: verseuchte Böden, Schrott, Kunststoffe und explosive Schrittmacher.

Die Finissage verspricht andere intermortale Zustände mit Überraschungen am Samstag, dem
1. April 2006. Bis es soweit ist, sorgen Kamingespräche, Führungen, Workshops, Film und Konzerte für die Lebenden. Auskünfte über Tel. 0434464469 oder www.museum-bellerive.ch. Als «dance macabre» seien einige Links aus dem lesenswerten Katalog zur Weiterbildung empfohlen: www.alcor.org, www.crazycoffins.co.uk, www.findagrave.com, www.friedwald.ch, www. grufti.de, www.leary.com, www.memorialspace flights.com, www.naturbestattungen.de, www. salon-funeraire.com und www.rattenfriedhof.de.

\title{
Über dem Grabe
}

\author{
E. Danieli
}

Korrespondenz:

Dr. med. Enrico Danieli

Via ai Colli 22

CH-6648 Minusio
Hebbels Tagebücher begleiten mich durch das Leben. Unterwegs blätternd bleibe ich stehen und haften an seinen unzeitgemässen Gedanken: «Nur nicht lange leben und nicht lange sterben, das übrige ist gleich. Das Leben ist ein Verbrennungsprozess: Ein trübes Dasein ist wie ein Scheiterhaufen, der angezündet wird, während es regnet.» Jedesmal wenn die schwarzäugige, jugendlich gebliebene Portugiesin, Lingeriemitarbeiterin seit Jahrzehnten, mich besucht, zeigt sie mir, abgemacht und stumm, unaufgefordert und erwartet und beinahe ein wenig ritualhaft die vergilbte Photographie ihres einzigen, früh verstorbenen Kindes: Ein Mädchen im späten Säuglingsalter, schwarzhaarig, schwarzäugig, dreht auf einem ehemals bunten Kinderkarussell tief im Süden Europas Runden. Es thront, nur mit Mühe und mit Hilfe der mitfahrenden Mutter mit Händchen sich am Bügel festhaltend, auf einem Engelskopf: wallendes, üppiges, weisses Haar, weit von sich gespreizte Flügel, goldene, träumerische Augen. Ich kann es nicht erklären, aber dieser Tod verfolgt mich, obwohl ich das Kind nicht kenne, nie gekannt habe, nur vom späteren Schicksal der Mutter, der Portugiesin, weiss: ledig, ausgestossen, zeitlebens verdammt. Eine nie endende und jeder Therapie trotzende Demütigung.

Das «sudden infant death syndrome», der plötzliche und unerwartete Kindstod, verlange nach einer Autopsie. Dies ist meine hilflose Erklärung für zusätzlich erlittenes Leid. Hier kam zum Todesgrauen noch die medizinische Traumatisierung hinzu: Untersuchungsrichter, Be- zirksanwalt, Rechtsmediziner, Polizei, Schuldzuweisungen, Kinderarzt, Pfarrer, Verwandte, Aufbahrungshalle, Beerdigung, Erd- oder Feuerbestattung, Sargrückführung.

Ein Tod ohne Krankheit, wie wir leichthin sagen, obwohl, wie von Spezialisten dagegengehalten, terminal, also noch vor dem Tod, durchaus Lungenblutungen, Hirnschwellungen, Blutleere im Bauchraum und in Präparaten, vielfach gezeigt, früh- oder vorzeitige Selbstauflösungen der Darmschleimhaut nachweisbar seien. Doch wer, fragen wir, löst die Störungen aus, wann und warum greifen diese in das Lebensgeschehen ein, und was wird mit diesen Todespräparaten schon bewiesen? Bilder, Schicht-Klapp-Bilder, Modelle, Präparate vermitteln früh schon Kenntnisse toter Mütter, toter Föten, toter Kinder, bald ermöglichen neue Präparierungstechniken die Darstellung der menschlichen Embryonalentwicklung, des ganzen Menschen: Reliefs aus gebranntem Ton, Wachspräparate, Elfenbeinskulpturen. Heute sind es Ganzkörperplastinate, die den Tod anschaulich, verständlich, verstehbar machen.

Doch warum macht das Sterben in den ersten Lebensmonaten genauso betroffen wie einst? Ist es die Unschuld der Kinder, die vom Tod nicht respektiert wird, ist es unsere uns quälende Unwissenheit, die den Tod wie stets zum uns Überlegenen macht? Wie sagt doch Hebbel: «Der Tod begeht keinen Fehler, wenigstens macht er keinen wieder gut.» Früher, wir erfahren es eindrücklich in der medizinhistorischen Ausstellung «Über dem Grabe geboren», war der 
Anteil der Kinder unter den Verstorbenen erschreckend hoch. Doch falsch ist die Vorstellung, wegen des damaligen Kinderreichtums sei keine emotionale Bindung möglich gewesen. Obwohl das Wissen um den allgegenwärtigen Tod stets vorhanden war, hatten Eltern und Geschwister, wie entsprechende Zeugnisse beweisen, ausgeprägte Gefühle gegenüber dem unzeitgemäss hingegangenen Kind: Totentänze, Totenporträts, Kinderbegräbnisse, Spielgefährten versammeln sich um das Totenbett, um das Engelein zu sehen, Schutzengel, in Grabdarstellungen, begleiten die toten Kinder in das Paradies. Denn tote Kinder sind Engel: Wenn ein Kind stirbt, redet man häufig von einem Engelsbegräbnis, und Friedhöfe kennen auch heute eigene Abteilungen für Kinderleichen: Engelgärtlein.

Anstelle der christlich-kirchlich-religiösen Rituale mit Fürbitten, Armenseelenkult, Gnadesgewissheit, Trauergottesdiensten, Totenwachen, Grabpflege wird der Tod heute ästhetisiert: Totenbildnisse, Photographien sind Ausdruck vom Wunsch nach Festhalten der Erinnerung. Doch geblieben ist das Entsetzen über ein Geschehen, das heute wie damals unerklärbar ist: «... den 14. Feb. 1847: abends 9 Uhr ist der Engel gestorben, ohne vorher krank gewesen zu sein ... am Herzkrampf, wie der Arzt sagte ... das Kind schrie heftig, es hatte die Augen im Kopf verdreht ... ich ahnte nicht, dass der Zufall etwas Schlimmes bedeuten könne ... das Kind hatte zu atmen aufgehört ... ich will nicht mit meiner Lebensphilosophie in Widerspruch treten, ich habe einen raschen Tod immer für den besten erklärt, und er ist es, aber nur für den Sterbenden selbst ...»

Genau in diesem Sinne heute mit der Portugiesin zu sprechen versucht. Doch sie hat mich entgeistert angeschaut, hat den Kopf geschüttelt und wiederholt und wie abwesend mit den Fingern über die Photographie gestrichen. Ich hoffe sehr, dass meine Erklärungsversuche nicht unser Ritual des gemeinsamen Fotobetrachtens beendet haben. 